\title{
ANÁlISE DIRETA DE SÓLIDOS POR ESPECTROMETRIA DE ABSORÇÃo ATÔMICA COM ATOMIZAÇÃO EM FORNO DE GRAFITE: UMA REVISÃO
}

\author{
Cassiana Seimi Nomura \\ Centro de Ciências Naturais e Humanas, Universidade Federal do ABC, Rua Santa Adélia, 166, 09210-170 \\ Santo André - SP, Brasil \\ Cíntia Soares da Silva e Pedro Vitoriano Oliveira* \\ Instituto de Química, Universidade de São Paulo, CP 26077, 05513-970 São Paulo, SP - Brasil
}

Recebido em 11/7/06; aceito em 30/3/07; publicado na web em 19/12/07

\begin{abstract}
SOLID SAMPLING GRAPHITE FURNACE ATOMIC ABSORPTION SPECTROMETRY: A REVIEW. This is a review of direct analysis using solid sampling graphite furnace atomic absorption spectrometry. Greater emphasis is dedicated to sample preparation, sample homogeneity, calibration and its application to microanalysis and micro-homogeneity studies. The main advantages and some difficulties related to the applicability of this technique are discussed. A literature search on the application of solid sampling graphite furnace atomic absorption spectrometry in trace element determination in many kinds of samples, including biological, clinical, technological and environmental ones, is also presented.
\end{abstract}

Keywords: solid sampling; atomic absorption spectrometry; graphite furnace.

\section{INTRODUÇÃO}

A maioria das amostras submetidas para determinação elementar por espectrometria de absorção atômica com forno de grafite (GF AAS) encontra-se na forma sólida. Usualmente, as amostras são convertidas em soluções aquosas por meio de procedimentos clássicos por via seca, tais como, cinzas para materiais orgânicos, fusão para materiais inorgânicos refratários e por via úmida, digestão ou dissolução ácida em altas temperaturas para amostras orgânicas e inorgânicas, repectivamente ${ }^{1-3}$. Esses procedimentos apresentam características favoráveis e limitações que dependem, particularmente, do tipo de amostra, dos analitos e de suas concentrações. Erros sistemáticos devido à contaminação ou perdas por volatilização estão entre os mais freqüentes, podendo afetar diretamente a exatidão e precisão dos resultados analíticos. Considerando que a GF AAS é uma técnica destinada à determinação de traços e ultratraços de elementos, os cuidados no preparo das amostras tornam-se cruciais para o sucesso do método empregado ${ }^{4-6}$.

Nesse contexto, a determinação elementar a partir da análise direta da amostra sólida, com o mínimo de manipulação é uma alternativa bastante viável. A análise direta de sólidos, que pode ser efetuada por meio de suspensões ou por meio da amostragem direta de uma amostra pulverizada, com tamanho de partícula controlado, apresenta algumas vantagens quando comparada aos procedimentos convencionais $^{6,7}$ : simplificação no pré-tratamento da amostra, possibilitando reduzir o tempo gasto nessa etapa, aumentando, assim, a frequiência analítica; minimização dos riscos de contaminação devido ao uso de quantidades reduzidas de reagentes, pouca manipulação ou baixa exposição ao ambiente; minimização das perdas do analito de interesse; menor periculosidade devido a não utilização de reagentes tóxicos ou corrosivos, por exemplo, HF; minimização de geração de resíduos, o que permite classificar os métodos como "limpos"; maior detectabilidade, uma vez que as amostras não são diluídas e, possibilidade de analisar uma pequena quantidade de amostra, permitindo estudos de micro homogeneidade.

*e-mail: pvolivei@iq.usp.br
A GF AAS apresenta características que a elegem como uma das técnicas mais adequadas para análise direta de sólidos, seja na forma de suspensão (SiS GF AAS) ou de sólido direto (SS GF AAS) $)^{4-11}$. Dentre as suas principais características, destacam-se: o programa de aquecimento que permite o pré-tratamento térmico da amostra, sobretudo durante a etapa de pirólise, o que facilita a remoção de parte dos concomitantes que podem provocar interferências durante a atomização; as pequenas quantidades de amostras que podem ser analisadas; apresenta boa seletividade e sensibilidade; os problemas relacionados ao sistema de transporte são praticamente inexistentes, uma vez que o transporte das suspensões é feito por amostragem discreta (não depende de nebulizadores), e os sólidos são pesados diretamente nas plataformas de grafite que são, posteriormente, introduzidas no atomizador eletrotérmico e, a adequada otimização do programa de aquecimento, aliada ao uso de modificadores químicos, possibilita, na maioria das vezes, a calibração do equipamento com soluções aquosas.

\section{SS GF AAS: CONTEXTO HISTÓRICO}

Historicamente, a SS GF AAS é quase tão antiga quanto a própria técnica de espectrometria de absorção atômica. Boris L' Vov foi um dos pioneiros, quando testou o desempenho do seu modelo de forno de grafite após amostragem direta de cloreto de sódio para análise elementar ${ }^{12}$. O primeiro forno de grafite idealizado por L' Vov apresentava uma configuração que era bastante adequada para amostragem direta de sólidos. Porém, na simplificação do modelo, posteriormente proposta por Massmann ${ }^{13}$, e que foi aquela adotada pela maioria dos fabricantes, a amostragem direta de sólidos não era tão trivial.

Ao longo do desenvolvimento instrumental da técnica, diversos acessórios e modificações nos fornos surgiram na tentativa de facilitar e permitir a análise direta de sólidos ${ }^{4,7,8}$. Em geral, os modelos de amostradores propostos visavam a introdução de amostra sólida em um atomizador empregado para amostragem de soluções.

Apesar do interesse na análise direta de sólidos por GF AAS, as pesquisas que culminaram em um modelo de forno de grafite designa- 
do para essa finalidade se intensificaram a partir da década de $80^{4-8}$. Dois fatores podem ser atribuídos a esse crescente interesse: o surgimento dos fornos de grafite com aquecimento transversal e o uso de corretores de radiação de fundo baseados no efeito Zeeman, que para a maioria dos elementos apresenta melhor eficiência que os corretores com lâmpada de deutério. Além disso, na SS, por causa da ausência da diluição da amostra, os componentes da matriz no forno também estão mais concentrados, levando a sinais de fundo em geral maiores, que podem requerer corretores mais eficientes.

Atualmente, encontra-se comercialmente disponível um modelo de espectrômetro de absorção atômica com atomização eletrotérmica que opera no modo convencional, com solução aquosa, ou com amostragem direta de sólidos. Nesse equipamento, a amostra sólida é pesada diretamente em uma plataforma de grafite pirolítico do tipo "canoa", que é inserida lateralmente no interior do tubo de grafite com auxílio de uma pinça ${ }^{8,14}$. Esses amostradores podem ser muito simples, com operação manual ou mais sofisticados, com balança e pinça operadas automaticamente ${ }^{8}$.

Apesar das características favoráveis e dos excelentes resultados em matrizes diversas, pode-se considerar que o número de aplicações da SS GF AAS ainda é pequeno. Na Figura 1 estão apresentados os números de artigos publicados, desde 1973, relacionados à análise de sólidos por GF AAS. O perfil do gráfico apresentado na figura indica que a técnica teve um aumento no número de trabalhos publicados a partir dos anos 90. É interessante notar que, a partir de 1995, o número de publicações vem se mantendo constante, com nítida tendência de crescimento a partir de 2003. Como mencionado anteriormente, esse crescente interesse deve estar relacionado à melhoria na instrumentação disponível comercialmente, que permitiu ampliar a capacidade de aplicações da técnica. O lançamento recente de um espectrômetro de absorção atômica de alta resolução com fonte contínua (HR-CS AAS) ${ }^{15}$, com capacidade única de correção de fundo, de visualização espectral e possibilidade de determinação multielementar a partir da amostragem direta de sólidos, deverá impulsionar as pesquisas e a popularização da técnica.

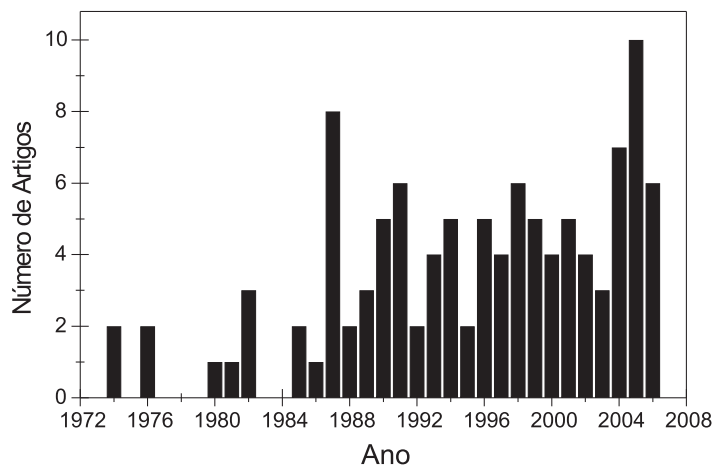

Figura 1. Número de artigos publicados por ano referentes à SS ETAAS

Não obstante as vantagens referentes à SS GF AAS, algumas dificuldades ainda precisam ser superadas e que, dependendo da amostra, podem ser um obstáculo para aplicação em análise de rotina: a calibração do equipamento; a homogeneidade da amostra e, a representatividade devido às pequenas massas de amostra que são analisadas.

\section{PREPARO DA AMOSTRA PARA SS GF AAS}

Apesar de dispensar os morosos pré-tratamentos das amostras, na análise direta de sólidos por SS GF AAS é recomendável que as amostras sejam secas e moídas até se transformarem em um pó muito fino com tamanho de partículas controlado. No entanto, há trabalhos na literatura propondo a introdução direta de pedaços de polímeros ${ }^{16}$ e materiais biológicos como sangue, fígado e rim $^{17}$ in natura para serem analisados.

A secagem deve ser realizada, preferencialmente, por métodos que não interfiram nas propriedades físicas e químicas das amos$\operatorname{tras}^{18}$. A liofilização é um processo comumente empregado para secar amostras biológicas e tem sido também utilizada como uma técnica quantitativa de pré-concentração de elementos traço em águas ${ }^{19}$. Jatos de ar aquecido $\left(80\right.$ a $85{ }^{\circ} \mathrm{C}$ ) também podem ser utilizados na secagem de amostras. Essa técnica foi empregada na secagem de fígado de porco visando a preparação de material certificado ${ }^{20}$. Forno de microondas caseiro ( $30 \mathrm{~W}$ durante $45 \mathrm{~min}$ ) associado ao aquecimento em estufa $\left(60{ }^{\circ} \mathrm{C}\right.$ por aproximadamente $\left.48 \mathrm{~h}\right)$ também foi empregado, com sucesso, para secar amostras de fígado bovino ${ }^{21}$.

A homogeneização é um outro importante processo da etapa de preparo de amostra para análise direta de sólidos e é facilmente alcançada por procedimentos de moagem. Em geral, quanto mais estreita a faixa de distribuição do tamanho de partícula e menor o seu diâmetro $(<10 \mu \mathrm{m})$ melhor será a homogeneidade da amostra ${ }^{6,22}$.

A escolha do sistema de moagem a ser empregado pode variar dependendo das propriedades da amostra, tais como a dureza e o conteúdo de fibras e gorduras. A moagem criogênica, introduzida por Iyengar e Kasperek em $1977^{23}$, tem como princípio fundamental o aumento da dureza do material e inserção de falhas na estrutura cristalina, que facilitam o processo de moagem ${ }^{23,24}$. Desde a sua proposição, a moagem criogênica tem sido intensamente empregada para moer diferentes tipos de amostras ${ }^{21,25,26}$.

Outros moinhos mecânicos como de almofariz e pistilo, de blenda, de disco, de facas e de bolas têm sido aplicados para moer amostras. Um moinho de bolas foi empregado com sucesso na preparação do fígado suíno certificado ${ }^{20}$, amostras de peixe ${ }^{27}$ e fígado bovino $^{21}$. O uso de almofariz e pistilo também mostrou ser uma boa alternativa para moer amostras de peixe, visando a determinação de $\mathrm{Se}^{28}$.

Em um estudo desenvolvido por Fajgeli e Zeisler ${ }^{29}$, os tamanhos de partículas de diversos materiais de referências certificados do IAEA ("International Atomic Energy Agency", Viena, Áustria) e NIST ("National Institute of Standards and Tecnology", Gaithersburg, MD, USA) foram avaliados. Alguns desses materiais eram ambientais e biológicos, moídos em diferentes sistemas. Como conclusão, os autores observaram que os materiais biológicos moídos em moinhos de jato de ar foram os que apresentaram os menores tamanhos de partícula e, conseqüentemente, os mais adequados para serem empregados em microanálises.

\section{FATORES QUE INFLUENCIAM A ANÁLISE DIRETA DE SÓLIDOS POR SS GF AAS}

Considerando a quantidade de amostra que é tomada para análise, a SS GF AAS pode ser classificada como uma técnica em que se pratica desde a micro- até a submicroanálise, com massas que podem variar de 10 a $0,01 \mathrm{mg}$, respectivamente ${ }^{30,31}$.

Apesar da possibilidade de analisar massas diminutas de amostras ser uma das grandes vantagens da SS GF AAS, essa característica é também um grande desafio, pois obter resultados precisos e exatos nessas condições não é uma tarefa trivial. Em geral, massas inferiores a $100 \mathrm{mg}$ tendem a comprometer a homogeneidade e, consequientemente, a representatividade, já que os elementos traço podem não estar homogeneamente distribuídos pelo material ${ }^{32}$. Quando a amostra é subdividida em porções cada vez menores, ela tende a se tornar cada vez mais não homogênea, pois a probabilidade estatística de encontrar a mesma concentração média do analito na fração tomada para análise torna-se cada vez menor, vide repre- 
sentação apresentada na Figura 2. Em geral, massas de amostra de 100 a 500 mg são pesadas para digestão ou solubilização ${ }^{22}$. Nesses casos, a micro homogeneidade não é um fator limitante e não deve gerar erros associados à falta de homogeneidade da amostra (Figura 2a). Por outro lado, quando massas diminutas são tomadas para análise $(0,01 \mathrm{a} 1 \mathrm{mg})$ a micro homogeneidade torna-se um parâmetro crítico. As diferentes porções de amostras tomadas para análise podem conter diferentes quantidades do elemento de interesse (Figura 2b), gerando grande imprecisão entre as medidas e, desta forma, comprometendo os resultados analíticos.
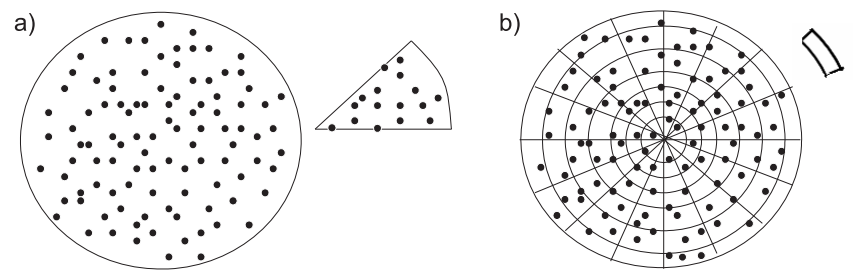

Figura 2. Diagrama para ilustrar a influência da homogeneidade na determinação de um analito $(\bullet)$ : (a) grande massa de amostra e (b) pequena massa de amostra

Quando um material homogêneo se torna heterogêneo para pequenas massas, existe uma massa mínima que deve ser considerada e bem estabelecida para garantir a representatividade da amostra e a exatidão dos resultados analíticos ${ }^{32}$. A influência da massa de amostra sobre a precisão dos resultados analíticos foi avaliada por Rossbach et $a$. $^{33}$, que verificaram que o desvio padrão relativo (RSD) das medidas diminuiu de 50 para $0,5 \%$ quando massas de amostras foram aumentadas de 0,01 para $100 \mathrm{mg}$. Langmyhr ${ }^{34}$ mostrou, em artigo de revisão, que para muitos materiais, massas entre 0,1 e $10 \mathrm{mg}$ podem ser analisadas sem comprometer a representatividade. Belarra et al..$^{35}$, por sua vez, demonstraram que massas muito elevadas também podem gerar resultados inexatos em SS GF AAS. Segundo esses autores, que visavam a determinação de $\mathrm{Cu}$ em complexo vitamínico, a análise de elevadas massas de amostra gerava resultados superestimados, provavelmente devido à influência da matriz que alterava a cinética de vaporização do analito.

A área superficial das partículas pode exercer grande influência sobre o processo de atomização ${ }^{36}$. Já foi demonstrado que quando sucessivos procedimentos de atomização são aplicados a uma amostra com partículas de forma esférica, o sinal de absorção desaparece somente após diversos ciclos de atomização.

Considerando a homogeneidade de materiais, pode-se dizer que a maioria dos sólidos, com raras exceções como algumas ligas metálicas e vidros, é uma mistura heterogênea. Materiais biológicos, geológicos e ambientais são caracteristicamente não homogêneos, sendo as rochas, os solos e sedimentos os materiais que apresentam composições mais heterogêneas ${ }^{5}$.

Portanto, a homogeneidade é um importante pré-requisito que deve ser considerado para a análise direta de amostras sólidas ${ }^{22}$. Esse parâmetro depende de fatores como a natureza do material, a densidade, a massa de amostra a ser utilizada e a concentração do elemento de interesse. Experiências têm mostrado que a heterogeneidade para pequenas massas de amostras é, geralmente, consequiência da presença de partículas grandes "aglomeradas", as quais podem conter concentrações de elementos em níveis de traço muito maiores que aquelas encontradas em toda a amostra ${ }^{37}$. Uma maneira de contornar esse tipo de problema é reduzir o tamanho das partículas das amostras por meio de procedimentos de moagem, como já anteriormente mencionado.

A homogeneização é uma das etapas mais importantes e críticas do processo de produção de materiais de referência certifica- dos (CRM). Esses materiais são muito importantes na Química Analítica e atuam como a principal fonte de avaliação da exatidão dos métodos propostos. Desse modo, é importante que as características desses materiais, entre elas a homogeneidade, sejam bem definidas. A maioria dos CRM atualmente disponíveis no mercado apresenta homogeneidade garantida somente para elevadas massas de amostras, variando entre 100 e $500 \mathrm{mg}^{22}$. Isso é um problema, pois técnicas que praticam a microanálise utilizam massas de amostra geralmente inferiores a $10 \mathrm{mg}$. Considerando a escassez de CRM para microanálise, alguns esforços vêm sendo direcionados no sentido de produzir materiais que atendam a essas exigências. Nesse sentido, avaliar a micro homogeneidade desses materiais torna-se uma etapa importante no processo de produção ${ }^{21,38}$. Atualmente existe disponível um material certificado de sedimento marinho (SRM 2703), produzido pelo NIST, que pode ser utilizado em microanálise, pois o mesmo apresenta homogeneidade garantida para massa de $0,7 \mathrm{mg}$.

\section{AVALIAÇÃO DA MICRO HOMOGENEIDADE}

De acordo com o Guia ISO $35^{39}$, um material é perfeitamente homogêneo com relação a uma determinada característica, se ao comparar duas partes distintas do mesmo material não houver diferença nos valores dessa característica. Na prática, um material pode ser considerado homogêneo com relação a uma característica se a diferença entre o valor de uma parte com relação à outra parte não puder ser identificada experimentalmente. $\mathrm{O}$ conceito básico de homogeneidade, portanto, engloba tanto a característica como o parâmetro da medida (geralmente o desvio padrão), incluindo a massa de amostra da porção testada.

Experimentalmente, o grau de homogeneidade pode ser determinado pela medida de uma propriedade (por exemplo, concentração de um elemento) em uma pequena porção (por exemplo, massa de amostra), utilizando um método com precisão relativamente elevada.

A técnica a ser utilizada para a avaliação da homogeneidade é outro fator importante. Segundo Pauwels et al. ${ }^{32}$, a escolha da técnica deve ser feita considerando alguns requisitos importantes: não deve requerer pré-tratamento da amostra para evitar perdas dos analitos e contaminações; deve possibilitar análise precisa de pequenas massas de amostras, preferencialmente menor que $1 \mathrm{mg}$ e, deve apresentar, preferencialmente, uma frequiência analítica alta. A SS GF AAS contempla todas essas características e, por isso, tem sido utilizada para avaliar a homogeneidade de diversos tipos de materiais ${ }^{21,38}$.

A informação a respeito de homogeneidade de amostras foi inicialmente proposta por Igamells e Switzer ${ }^{40}$ quando apresentaram a constante de amostragem $\left(\mathrm{K}_{\mathrm{S}}\right)$ para estimar a imprecisão devido ao erro de amostragem, Equação 1

$\mathrm{RSD}=\left(\mathrm{K}_{\mathrm{S}} / \mathrm{m}\right)^{1 / 2}$

De acordo com essa equação, $\mathrm{K}_{\mathrm{S}}$ pode ser estimada pelo RSD das medidas das sub-amostragens de massa $m$. A Equação 1 não considera os erros aleatórios provenientes dos procedimentos analíticos. Para que essas incertezas sejam devidamente consideradas na avaliação da homogeneidade, pode-se utilizar outro cálculo

$\mathrm{S}_{\mathrm{H}}{ }^{2}=\mathrm{RSD}^{2}-\Sigma(\text { outras incertezas })^{2}$

Essas incertezas são decorrentes da pesagem e erros instrumentais, como por exemplo, estabilidade da lâmpada de catodo oco e variações da temperatura do atomizador. Enquanto $\mathrm{K}_{\mathrm{S}}$ foi apresentada originalmente para estimar a homogeneidade de amostras geológicas, nas quais massas de amostras relativamente altas 
eram analisadas, Kurfürst et l. $^{41}$ introduziram o conceito de constante de homogeneidade $\left(H_{e}\right)$ para pequenas massas de amostras

$H_{e}=\mathrm{S}_{\mathrm{H}} * \mathrm{~m}^{1 / 2}$

Por meio da Equação 3, o erro de amostragem $\mathrm{S}_{\mathrm{H}}$ de uma determinada massa de amostra $m(\mathrm{em} \mathrm{mg}$ ) pode ser estimado diretamente a partir dos valores de RSD das medidas e dos erros aleatórios do procedimento analítico. Como a massa de amostra $m$ utilizada nessa avaliação é tipicamente $1 \mathrm{mg}, H_{e}$ representa a imprecisão devida ao erro de amostragem para uma unidade de massa (1 $\mathrm{mg})$. Quando o fator de homogeneidade é menor que $10,\left(H_{e}<10\right)$, o material pode ser considerado suficientemente homogêneo.

\section{CALIBRAÇÃo}

A calibração é, para a maioria das técnicas, um dos passos mais importantes para garantir a qualidade dos resultados analíticos. Encontrar um método adequado para a construção da curva analítica de calibração é preocupação importante que o analista deve ter, especialmente para a amostragem direta de sólidos ${ }^{42}$.

Em GF AAS o intervalo linear de calibração é reconhecidamente muito estreito, limitando, em alguns casos, a amostragem direta de sólidos. No entanto, o advento do corretor de radiação de fundo baseado no efeito Zeeman com três campos magnéticos e com diferentes intensidades permitiu uma "diluição instrumental", possibilitando a amostragem de maiores massas de amostra, aumentando a representatividade ${ }^{43,44}$.

A escolha do material a ser utilizado para a calibração depende fortemente do comportamento da matriz e do analito. Em geral, para minimizar interferências, o material usado para calibração deve ter composição idêntica ou a mais próxima possível do material que está sendo analisado ${ }^{6}$. Nesse contexto, os materiais de referência certificados (CRM) são bastante empregados na calibração. Pode-se pesar massas crescentes de um CRM ou massas iguais de um tipo de CRM com diferentes concentrações do analito. Em geral, se a composição do material usado para calibração for muito semelhante à da amostra, o efeito de matriz sobre o processo de vaporização/atomização, em ambos os casos, ocorre da mesma maneira. Atualmente existem disponíveis no mercado diversos tipos de CRM produzidos por diversas instituições, entre as quais, NIST, BCR ("Community Bureau of Reference", IRMM, Geel, Bélgica), NRC ("National Research Council Canada, Institute for Environmental Research and Technology", Otawa, Ontário, Canadá) e NIES ("National Institute for Environmental Studies, Japan Environment Agency", Yatabemachi, Japão).

Embora os CRM sejam intensamente utilizados em SS ET AAS, existem algumas limitações que devem ser consideradas: muitos materiais não são certificados para alguns elementos de interesse; na prática é muito difícil encontrar CRM com composição semelhante à da amostra e, a maioria dos CRMs comercialmente disponíveis apresenta homogeneidade garantida somente para massa de amostra muito superior àquela praticada em SS ET AAS.

O uso de soluções de referência é a forma mais comum, simples e barata para construir a curva analítica de calibração. Além disso, as soluções não apresentam problemas de homogeneidade. Porém, o seu emprego em técnicas de amostragem direta de sólidos nem sempre é possível. Em SS GF AAS, o sucesso da calibração aquosa depende fortemente de fatores que estão relacionados com os mecanismos de atomização dos elementos de interesse. Apesar da forte influência de matriz da amostra sólida, muitos trabalhos utilizaram soluções aquosas para a construção das curvas analíticas de calibração. Na maioria dos casos, isso foi possível devido a uma refinada oti- mização do programa de aquecimento, à utilização de modificadores químicos, gás de purga alternativo ou mistura oxidante para melhorar a separação analito/matriz durante a etapa de pirólise.

Os modificadores químicos são comumente utilizados na análise direta por SS GF AAS. A adição de uma alíquota de modificador químico em solução sobre a amostra sólida é a forma mais usual de utilizar esse tipo de substância. Como os modificadores químicos em solução são comumente preparados em meio ácido, para alguns elementos, e.g. $\mathrm{Cd} \mathrm{e} \mathrm{Pb}$, a adição sobre a amostra sólida promove a extração, facilitando a interação entre o analito e o modificador ${ }^{45}$. Modificadores químicos permanentes como Ir, Pd, Rh, Ru e W também vêm sendo empregados, com sucesso, nas determinações por SS GF AAS ${ }^{21,46,47}$. Os mecanismos de ação desses modificadores sobre os analitos das amostras sólidas são bastante complexos. A interação entre o modificador químico permanente e o analito deve ocorrer, provavelmente, na fase condensada. Nesse caso, os analitos migram da amostra sólida para a superfície do modificador durante a etapa de pirólise $^{46}$.

\section{LIMITE DE DETECÇÃO}

De acordo com a definição da IUPAC, o limite de detecção, expresso em termos de quantidade de analito $\mathrm{a}_{\mathrm{L}}$, está relacionado à menor resposta que pode ser detectada com razoável certeza por um dado método analítico ${ }^{6}$. Em geral, o limite de detecção é obtido com base nas medidas do branco analítico. Idealmente, a resposta do branco analítico pode ser obtida a partir de materiais que possuem composição matricial similar à amostra que está sendo analisada, porém, isento do analito de interesse. Efetuar as medições dos sinais analíticos em condições idênticas às das amostras, inclusive na presença da mesma matriz é importante, pois a matriz pode gerar interferências que vão refletir nos resultados analíticos.

Quando as amostras são líquidas, encontrar o branco analítico não é crítico. Nesse caso, um branco sintético composto pode ser preparado, por exemplo, misturando solventes e elementos majoritários presentes na matriz. Por outro lado, em análise direta de sólido, a obtenção desse branco não é trivial, pois o material a ser utilizado para prepará-lo não existe ou é difícil de ser preparado sinteticamente.

Muitos trabalhos na literatura fazem uso de diferentes ferramentas para calcular o limite de detecção associado às técnicas de análise direta de sólidos. A forma mais usual tem sido o cálculo do limite de detecção a partir de uma solução de referência e seu correspondente branco. Entretanto, esse tipo de aproximação dificilmente pode ser utilizado como um guia real da análise direta de sólido.

Uma alternativa seria medir o "branco da amostra", por meio da obtenção do sinal do branco a partir de uma amostra com mesma composição matricial, porém com concentração muito baixa do analito de interesse ${ }^{6}$. Na dificuldade de encontrar esse tipo de material, é possível substituí-los por outros materiais sintéticos. O $\mathrm{SiC}$ aquecido a $2200{ }^{\circ} \mathrm{C}$ para eliminar as impurezas, por exemplo, é um candidato a branco analítico. Da mesma maneira, outros materiais como o grafite e a celulose de grau analítico são algumas boas opções ${ }^{6}$.

Uma outra forma de medir o branco analítico é realizar as medidas da plataforma de grafite vazia. O sinal de absorbância obtido refere-se à "massa zero" $21,38,45$. Nessa situação, o desvio padrão do branco analítico é aquele proveniente do "ruído" instrumental. No caso das determinações envolvendo comprimentos de onda mais sensíveis, o desvio padrão está diretamente relacionado às contaminações aleatórias.

Conhecendo-se o valor do desvio padrão de 20 medidas do branco analítico $(\sigma)$, pode-se calcular a massa do analito referente a esse desvio $\left(\mathrm{a}_{\mathrm{L}}\right.$ ) pela equação $3 \sigma / S$, na qual $S$ é a inclinação da curva analítica de calibração. Por fim, o valor do limite de detecção (em 
concentração) pode ser obtido utilizando-se a equação $C_{L}=a_{L} / m_{\max }$, na qual $\mathrm{m}_{\max }$ se refere à máxima massa de amostra analisada ${ }^{45}$.

\section{APLICAÇÕES MAIS RECOMENDADAS DA SS GF AAS}

Considerando as principais características da SS GF AAS, podese dizer que as aplicações são fortemente recomendadas em alguns casos específicos ${ }^{7,11}$ : determinação de elementos em amostras de difícil solubilização; determinações de elementos em níveis de traço e ultratraço em amostras de alta pureza; em situações nas quais a disponibilidade da amostra é pequena e, para avaliar a micro homogeneidade de materiais.

$\mathrm{Na}$ Tabela 1 estão apresentadas as aplicações da SS GF AAS para determinações de elementos em matrizes orgânicas, sendo as biológicas e de alimentos, a maioria. Muitos dos artigos exploram o uso da SS GF AAS para avaliar a distribuição dos analitos nas amostras e a micro homogeneidade de CRM. Na Tabela 2 estão apresentados os trabalhos que visam as determinações de elementos em amostras inorgânicas que, muitas vezes, possuem elevada pureza ou são caracteristicamente de difícil decomposição. Na Tabela 3, estão reunidos os trabalhos que fazem uso da SS GF AAS para investigações ambientais.

\section{CONCLUSÕES}

Considerando as principais vantagens associadas às técnicas que permitem a determinação direta de sólidos, a SS GF AAS se mostra como uma importante ferramenta para a química analítica aplicada. A alta sensibilidade, a relativa facilidade de amostragem de sólidos e a possibilidade de analisar massas muito pequenas de amostras tornam a técnica recomendada para microanálise. Apesar das inúmeras características positivas associadas à SS GF AAS, algumas dificuldades ainda existem e necessitam ser superadas, tais como, a calibração, o branco analítico e a repetibilidade entre medidas. O caráter monoelementar é um desafio que pode ser superado ao operacionalizar a introdução de amostras sólidas em um espectrômetro de absorção atômica com detecção simultânea (SIMAAS). A não homogeneidade das sub-amostras tomadas para análise é um parâmetro que deve ser sempre considerado. Porém uma moagem eficiente permite boa precisão para massas de amostras inferiores a $1 \mathrm{mg}$. A falta de CRMs para microanálise também é um problema e que, finalmente, está sendo lentamente resolvido com o empenho de certos grupos de pesquisa e centros de metrologia, reconhecidamente competentes, na produção de materiais de referência.

Tabela 1. Aplicação da SS ETAAS na análise de materiais orgânicos

\begin{tabular}{|c|c|c|c|}
\hline Amostra & $\begin{array}{l}\text { Elementos } \\
\\
\text { Tec }\end{array}$ & $\begin{array}{l}\text { Comentários } \\
\text { blógico, alimentos e medicamento }\end{array}$ & Ref. \\
\hline Alimentos & $\mathrm{P}$ & $\begin{array}{l}\text { Calibração com solução aquosa } \\
\text { Modificador químico: } 5 \mu \mathrm{g} \mathrm{Pd}+5 \mu \mathrm{g} \mathrm{Ca}\end{array}$ & 48 \\
\hline $\begin{array}{l}\text { Amostras biológicas } \\
\text { em geral }\end{array}$ & $\mathrm{Hg}$ & $\begin{array}{l}\text { Calibração com solução aquosa } \\
\text { Modificador químico: } 0,3 \mathrm{mg} \mathrm{KMnO}_{4}\end{array}$ & 49 \\
\hline Amostras biológicas & $\mathrm{Pb}$ & $\begin{array}{l}\text { HR-CS AAS } \\
\text { Modificador químico permanente: } 400 \mu \mathrm{g} \mathrm{Ru} \\
\text { Tamanho de partícula inferior a } 50 \mu \mathrm{m} \\
\text { Massa de amostra entre } 0,2 \text { e } 1,5 \mathrm{mg}\end{array}$ & 46 \\
\hline Amostras biológicas & $\mathrm{Cr}$ & $\begin{array}{l}\text { Calibração com adição de analito sobre } 1,0 \mathrm{mg} \text { de amostra sólida } \\
\text { Massa de amostra entre } 0,1 \mathrm{e} 1,2 \mathrm{mg}\end{array}$ & 50 \\
\hline $\begin{array}{l}\text { Amostras orgânicas } \\
\text { (complexo vitamínico, } \\
\text { arroz e PVC) }\end{array}$ & $\mathrm{Fe}, \mathrm{Mn}, \mathrm{Pb}$ & $\begin{array}{l}\text { Avaliação da distribuição dos elementos } \\
\text { Calibração com solução aquosa } \\
\text { Massa de amostra entre } 1 \text { e } 2 \mathrm{mg}\end{array}$ & 51 \\
\hline $\begin{array}{l}\text { Amostras variadas } \\
\text { (fígado bovino, solo, PP) }\end{array}$ & $\mathrm{Ag}$ & $\begin{array}{l}\text { Calibração com solução aquosa } \\
\text { Modificador químico: } 1 \mu \mathrm{g} \operatorname{Pd}\left(\mathrm{NO}_{3}\right)_{2}\end{array}$ & 44 \\
\hline Cabelo & $\mathrm{Cd}, \mathrm{Cr}, \mathrm{Pb}$ & $\begin{array}{l}\text { Calibração com solução aquosa } \\
\text { Massa de amostra entre } 0,2 \text { e } 4,0 \mathrm{mg}\end{array}$ & 52 \\
\hline Cabelo & As & $\begin{array}{l}\text { Distribuição de As ao longo do fio de cabelo } \\
\text { Modificador químico: } 30 \mu \mathrm{g} \mathrm{Pd}\left(\mathrm{NO}_{3}\right)_{2}+20 \mu \mathrm{g} \mathrm{Mg}\left(\mathrm{NO}_{3}\right)_{2} \\
\text { Análise de segmentos de } 0,5 \mathrm{~mm} \text { de cabelo }\end{array}$ & 53 \\
\hline $\begin{array}{l}\text { Cálculo renal e } \\
\text { salivar }\end{array}$ & $\mathrm{Cd}, \mathrm{Cr}, \mathrm{Hg}, \mathrm{Ni}, \mathrm{Pb}$ & $\begin{array}{l}\text { Calibração com CRM } \\
\text { Massa de amostra entre } 0,1 \text { e } 5,0 \mathrm{mg}\end{array}$ & 54 \\
\hline $\begin{array}{l}\text { Cálculo salivar } \\
\text { humano }\end{array}$ & $\mathrm{Cd}, \mathrm{Pb}$ e $\mathrm{Zn}$ & $\begin{array}{l}\text { Moagem da amostra em moinho vibratório de ágata } \\
\text { Calibração com solução aquosa } \\
\text { Massa de amostra entre } 0,2 \text { e } 10 \mathrm{mg}\end{array}$ & 55 \\
\hline $\begin{array}{l}\text { Couve de bruxela, } \\
\text { Farinha de arroz, } \\
\text { Farinha de trigo, } \\
\text { Fígado bovino, } \\
\text { Leite em pó, } \\
\text { Músculo bovino, } \\
\text { Peixe, Rim suíno }\end{array}$ & $\mathrm{Cd}, \mathrm{Pb}$ & $\begin{array}{l}\text { Avaliação da homogeneidade } \\
\text { Calibração com solução aquosa e com CRM } \\
\text { Massa de amostra entre } 0,04 \text { e } 5,0 \mathrm{mg}\end{array}$ & 56 \\
\hline Fígado bovino & $\mathrm{Cd}, \mathrm{Pb}$ & Produção de CRM; calibração com solução aquosa & 57 \\
\hline Fígado bovino & $\mathrm{Cd}, \mathrm{Pb}$ & $\begin{array}{l}\text { Análise de fígado fresco } \\
\text { Calibração com solução aquosa e com CRM } \\
\text { Massa de amostra entre } 0,030 \text { e } 10 \text { mg }\end{array}$ & 58 \\
\hline
\end{tabular}


Tabela 1. continuação

\begin{tabular}{|c|c|c|c|}
\hline Amostra & Elementos & $\begin{array}{l}\text { Comentários } \\
\text { Tecido biológico e alimentos }\end{array}$ & Ref. \\
\hline Fígado bovino & $\mathrm{Cd}, \mathrm{Pb}$ & $\begin{array}{l}\text { Avaliação da homogeneidade } \\
\text { Calibração com solução aquosa } \\
\text { Modificador químico: } 250 \mu \mathrm{g} \mathrm{W}+200 \mu \mathrm{g} \mathrm{Rh} \\
\text { Massa mínima de amostra: } 0,04 \mathrm{mg}(\mathrm{Cd}) \text { e } 1,4 \mathrm{mg}(\mathrm{Pb})\end{array}$ & 38 \\
\hline Fígado bovino & $\mathrm{Cd}, \mathrm{Cu}, \mathrm{Pb}, \mathrm{Zn}$ & Calibração com CRM; Massa de amostra entre 0,5 e $5,0 \mathrm{mg}$ & 59 \\
\hline Fígado bovino & $\mathrm{Cd}, \mathrm{Cu}, \mathrm{Co}, \mathrm{Fe}, \mathrm{Pb}, \mathrm{Zn}$ & $\begin{array}{l}\text { Calibração com solução aquosa } \\
\text { Modificador químico: }\left(\mathrm{NH}_{4}\right)_{2} \mathrm{SO}_{4} \\
\text { Massa de amostra entre } 0,2 \text { e } 2,0 \mathrm{mg}\end{array}$ & 60 \\
\hline Fígado bovino & $\mathrm{Cu}, \mathrm{Zn}$ & $\begin{array}{l}\text { Avaliação da homogeneidade } \\
\text { Calibração com solução aquosa } \\
\text { Modificador químico: } 250 \mu \mathrm{g} \mathrm{W}+200 \mu \mathrm{g} \mathrm{Rh} \\
\text { Massa mínima de amostra: } 0,04 \mathrm{mg}(\mathrm{Cu}) \text { e } 0,03 \mathrm{mg}(\mathrm{Zn})\end{array}$ & 21 \\
\hline $\begin{array}{l}\text { Fígado bovino, dente, } \\
\text { osso, músculo, } \\
\text { sangue, córtex renal }\end{array}$ & $\mathrm{Cd}, \mathrm{Pb}$ & $\begin{array}{l}\text { Avaliação da homogeneidade } \\
\text { Calibração com solução aquosa } \\
\text { Produção de material de referência }\end{array}$ & 61 \\
\hline Fígado eqüino & $\mathrm{Cd}, \mathrm{Pb}$ & $\begin{array}{l}\text { Avaliação da distribuição dos elementos } \\
\text { Calibração com solução aquosa }\end{array}$ & 62 \\
\hline $\begin{array}{l}\text { Fígado de pato } \\
\text { selvagem }\end{array}$ & $\mathrm{Cd}, \mathrm{Pb}, \mathrm{Hg}$ & $\begin{array}{l}\text { Avaliação da homogeneidade } \\
\text { Calibração com solução aquosa } \\
\text { Modificador químico: } \mathrm{NH}_{4} \mathrm{H}_{2} \mathrm{PO}_{4} \\
\text { Massa de amostra entre } 0,1 \text { e } 10 \mathrm{mg}(\mathrm{Cd}, \mathrm{Pb}) \text { e } 1 \text { e } 100 \mathrm{mg}(\mathrm{Hg})\end{array}$ & 63 \\
\hline $\begin{array}{l}\text { Fígado de pato } \\
\text { selvagem }\end{array}$ & $\mathrm{Cd}, \mathrm{Pb}$ & $\begin{array}{l}\text { Avaliação da distribuição dos elementos } \\
\text { Calibração com solução aquosa } \\
\text { Massa de amostra entre } 0,18 \text { e } 6,5 \mathrm{mg}\end{array}$ & 64 \\
\hline Folha de tabaco & $\mathrm{Cd}, \mathrm{Co}, \mathrm{Ni}, \mathrm{Pb}$ & $\begin{array}{l}\text { Modificador químico: } \mathrm{PdCl}_{2}+\mathrm{Pd}\left(\mathrm{NO}_{3}\right)_{2} \\
\text { Calibração com solução aquosa } \\
\text { Tamanho de partículas } \sim 20 \mu \mathrm{m}\end{array}$ & 65 \\
\hline Folha de tomate e chá & $\mathrm{Cu}$ & $\begin{array}{l}\text { Avaliação dos métodos de calibração } \\
\text { Calibração com material sólido sintético } \\
\text { Massa de amostra entre } 0,1 \text { e } 1,5 \mathrm{mg}\end{array}$ & 66 \\
\hline Frutos do mar & $\mathrm{Hg}, \mathrm{Cd}, \mathrm{Mn}, \mathrm{Pb}$ e $\mathrm{Sn}$ & $\begin{array}{l}\text { Calibração com CRM } \\
\text { Massa da amostra inferior a } 1 \mathrm{mg}\end{array}$ & 67 \\
\hline $\begin{array}{l}\text { Materiais biológicos } \\
\text { em geral }\end{array}$ & $\mathrm{Al}, \mathrm{Ag}, \mathrm{Cu}, \mathrm{Mn}, \mathrm{Pb}$ & $\begin{array}{l}\text { Calibração com solução aquosa } \\
\text { Massa de amostra entre } 1 \text { e } 4 \text { mg }\end{array}$ & 68 \\
\hline Músculo de bacalhau & $\mathrm{Pb}, \mathrm{Cd}, \mathrm{Hg}, \mathrm{Fe}, \mathrm{Zn}$ & $\begin{array}{l}\text { Verificação da micro homogeneidade } \\
\text { Calibração com CRM } \\
\text { Massa de amostra entre } 0,79 \text { e } 2,47 \mathrm{mg}\end{array}$ & 69 \\
\hline Músculo bovino & $\mathrm{Pb}$ & Massa de amostra entre 1,0 e $10,0 \mathrm{mg}$ & 70 \\
\hline Músculo eqüino & $\mathrm{Cd}$ & $\begin{array}{l}\text { Calibração com solução aquosa } \\
\text { Massa de amostra entre } 0,1 \text { e } 5,0 \mathrm{mg}\end{array}$ & 71 \\
\hline $\begin{array}{l}\text { Músculo de pato } \\
\text { selvagem }\end{array}$ & $\mathrm{Pb}$ & $\begin{array}{l}\text { Calibração com solução aquosa } \\
\text { Modificador químico: } \mathrm{NH}_{4} \mathrm{H}_{2} \mathrm{PO}_{4} \\
\text { Massa de amostra entre } 0,04 \text { e } 5,0 \mathrm{mg}\end{array}$ & 72 \\
\hline Peixe (bacalhau) & $\mathrm{Cd}$ & $\begin{array}{l}\text { Investigação da calibração } \\
\text { Calibração com CRM } \\
\text { Massa de amostra entre } 0,02 \text { e } 0,12 \mathrm{mg}\end{array}$ & 73 \\
\hline Pena de ave & $\mathrm{Pb}, \mathrm{Cd}$ & $\begin{array}{l}\text { Avaliação da distribuição dos elementos } \\
\text { Massa de amostra entre } 0,050 \text { e } 1,000 \mathrm{mg}\end{array}$ & 74 \\
\hline Planta & $\mathrm{Cr}, \mathrm{Mn}$ & $\begin{array}{l}\text { Calibração com solução aquosa } \\
\text { Massa de amostra entre } 0,2 \text { e } 4,0 \mathrm{mg}\end{array}$ & 52 \\
\hline Rim de ave & $\mathrm{Cd}, \mathrm{Pb}, \mathrm{Hg}$ & $\begin{array}{l}\text { Avaliação da distribuição dos elementos } \\
\text { Calibração com solução aquosa e com CRM } \\
\text { Massa de amostra entre } 0,039 \text { e } 0,090 \mathrm{mg}\end{array}$ & 75 \\
\hline Rim bovino & $\mathrm{Cd}, \mathrm{Pb}$ & $\begin{array}{l}\text { Avaliação da distribuição dos elementos } \\
\text { Calibração com solução aquosa } \\
\text { Massa de amostra entre } 0,7 \text { e } 8,0 \mathrm{mg}\end{array}$ & 76 \\
\hline Vegetais & $\mathrm{Cd}, \mathrm{Cu}, \mathrm{Ni}, \mathrm{Pb}$ & $\begin{array}{l}\text { Avaliação da homogeneidade } \\
\text { Calibração com solução aquosa } \\
\text { Massa de amostra entre } 0,1 \text { e } 2,0 \mathrm{mg}\end{array}$ & 77 \\
\hline
\end{tabular}


Tabela 1. continuação

\begin{tabular}{|c|c|c|c|}
\hline Amostra & Elementos & $\begin{array}{l}\text { Comentários } \\
\text { cido biológico e alimentos }\end{array}$ & Ref \\
\hline Vitamina B-12 & Co & Massa de amostra entre 0,1 e $2,0 \mathrm{mg}$ & 78 \\
\hline Vitaminas & $\mathrm{Mn}$ & $\begin{array}{l}\text { Calibração com solução aquosa e com CRM } \\
\text { Massa de amostra entre } 0,05 \text { e } 0,3 \mathrm{mg}\end{array}$ & 79 \\
\hline \multicolumn{4}{|c|}{ Polímeros } \\
\hline PET e PP & $\mathrm{P}$ & $\begin{array}{l}\text { Calibração com solução aquosa } \\
\text { Modificador químico: } 20 \mu \mathrm{g} \mathrm{Pd}\left(\mathrm{NO}_{3}\right)_{2}+20 \mu \mathrm{g} \text { ácido ascórbico } \\
\text { Massa de amostra: } 1,0 \text { e } 4,5 \mathrm{mg}\end{array}$ & 16 \\
\hline Plástico & $\mathrm{Cd}$ & $\begin{array}{l}\text { Avaliação da homogeneidade } \\
\text { Calibração com CRM } \\
\text { Massa de amostra entre } 0,060 \text { e } 0,250 \mathrm{mg}\end{array}$ & 80 \\
\hline Polietileno & & $\begin{array}{l}\text { Avaliação da homogeneidade e certificação } \\
\text { Massa de amostra entre } 0,060 \text { e } 0,250 \mathrm{mg}\end{array}$ & 81 \\
\hline Poliamida & $\mathrm{Si}$ & $\begin{array}{l}\text { Modificador químico: } 20 \mu \mathrm{g} \mathrm{Pd}\left(\mathrm{NO}_{3}\right)_{2} \text { ou } 1 \mu \mathrm{g} \mathrm{H}_{2} \mathrm{PtCl}_{6} \\
\text { Calibração com solução aquosa } \\
\text { Massa de amostra entre } 10 \text { e } 12 \mathrm{mg}\end{array}$ & 82 \\
\hline PVC & Sn & $\begin{array}{l}\text { Modificador químico: } \mathrm{Pd}\left(\mathrm{NO}_{3}\right)_{2} \\
\text { Calibração com solução aquosa }\end{array}$ & 83 \\
\hline PVC & $\mathrm{Sb}$ & $\begin{array}{l}\text { Calibração com solução aquosa } \\
\text { Massa de amostra entre } 2,5 \text { e } 3,5 \mathrm{mg}\end{array}$ & 84 \\
\hline
\end{tabular}

Tabela 2. Aplicação da SS ETAAS na análise de materiais inorgânicos

\begin{tabular}{|c|c|c|c|}
\hline Amostra & Elementos & Comentários & Ref \\
\hline Barita & $\begin{array}{l}\mathrm{As}, \mathrm{Cd}, \mathrm{Cu}, \mathrm{Cr} \\
\quad \mathrm{Ni}, \mathrm{Pb} \text { e V }\end{array}$ & $\begin{array}{l}\text { Adição de } 5 \mu \mathrm{L} \text { de } \mathrm{HNO}_{3} \text { concentrado } \\
\text { Calibração com solução aquosa } \\
\text { Massa de amostra entre } 0,04 \text { e } 0,7 \mathrm{mg}\end{array}$ & 85 \\
\hline Carbeto de silício & $\begin{array}{l}\mathrm{Al}, \mathrm{As}, \mathrm{Ca}, \mathrm{Cd}, \mathrm{Co}, \\
\mathrm{Cr}, \mathrm{Cu}, \mathrm{Fe}, \mathrm{K}, \mathrm{Li} \\
\mathrm{Mg}, \mathrm{Na}, \mathrm{Ni} \text { e Pb }\end{array}$ & $\begin{array}{l}\text { Tamanho de partículas inferiores a } 20 \mu \mathrm{m} \\
\text { Calibração com solução aquosa } \\
\text { Massa de amostra ente } 0,1 \text { e } 8 \mathrm{mg}\end{array}$ & 86 \\
\hline Cimento & $\mathrm{Cd}, \mathrm{Pb}$ e $\mathrm{Cr}$ & $\begin{array}{l}\text { Calibração com solução aquosa } \\
\text { Sistema de correção de BG 2-D } \\
\text { Massa de amostra entre } 0,020 \text { e } 0,400 \mathrm{mg}\end{array}$ & 87 \\
\hline Cobre & $\mathrm{Ag}$ & $\begin{array}{l}\text { Calibração com Ag metálica } \\
\text { Massas de amostra entre } 0,25 \text { e } 1,0 \mathrm{mg}\end{array}$ & 88 \\
\hline $\begin{array}{l}\text { Filtros de ar } \\
\text { (discos circulares) }\end{array}$ & $\mathrm{Cd}$ & Os filtros foram cortados e moídos em moinho de titânio & 89 \\
\hline $\begin{array}{l}\text { Fluoreto de cálcio e } \\
\text { outras amostras } \\
\text { contendo fluoretos }\end{array}$ & $\mathrm{Cu}, \mathrm{Cd}, \mathrm{Fe}, \mathrm{Pb}$ e $\mathrm{Zn}$ & $\begin{array}{l}\text { Diluição das amostras com carbono de alta pureza } \\
\text { Calibração com solução aquosa } \\
\text { Tamanho das partículas entre } 12 \text { e } 15 \mu \mathrm{m} \\
\text { Massas de amostra entre } 0,04 \text { e } 1,4 \mathrm{mg}\end{array}$ & 90 \\
\hline Grafite de alta pureza & $\begin{array}{l}\mathrm{Al}, \mathrm{As}, \mathrm{Ca}, \mathrm{Cd}, \mathrm{Co} \\
\mathrm{Cr}, \mathrm{Cu}, \mathrm{Fe}, \mathrm{K}, \mathrm{Li} \\
\mathrm{Mg}, \mathrm{Na}, \mathrm{Ni} \text { e } \mathrm{Pb}\end{array}$ & $\begin{array}{l}\text { Calibração com solução aquosa } \\
\text { Tamanho de partículas inferiores a } 20 \mu \mathrm{m} \\
\text { Massa de amostra ente } 0,1 \text { e } 8 \mathrm{mg}\end{array}$ & 91 \\
\hline Liga de alumínio & $\mathrm{Ga}$ & $\begin{array}{l}\text { Calibração com solução aquosa e com CRM } \\
\text { Massa de amostra ente } 0,05 \text { e } 5 \mathrm{mg}\end{array}$ & 92 \\
\hline $\begin{array}{l}\text { Ligas metálicas com } \\
\text { alto teor de } \mathrm{Ni}\end{array}$ & $\mathrm{Tl}, \mathrm{Bi}, \mathrm{Se}$ e Te & Calibração com solução aquosa & 93 \\
\hline Materiais cerâmicos & $\mathrm{Cu}, \mathrm{Ni}, \mathrm{Pb}$ e $\mathrm{Zn}$ & Calibração com solução aquosa & 94 \\
\hline $\begin{array}{l}\text { Materiais refratários } \\
\text { de alta pureza }\end{array}$ & $\begin{array}{l}\mathrm{Ca}, \mathrm{Fe}, \mathrm{K}, \mathrm{Mg} \\
\mathrm{Mn}, \mathrm{Na} \text { e } \mathrm{Zn}\end{array}$ & Calibração com solução aquosa & 95 \\
\hline Óxido de alumínio & $\begin{array}{l}\mathrm{Co}, \mathrm{Cr}, \mathrm{Cu}, \mathrm{Fe}, \mathrm{K} \\
\mathrm{Mg}, \mathrm{Mn}, \mathrm{Ni} \text { e } \mathrm{Zn}\end{array}$ & $\begin{array}{l}\text { Calibração com solução aquosa } \\
\text { Uso de metano como modificador químico } \\
\text { Tamanho de partículas inferiores a } 5 \mu \mathrm{m}\end{array}$ & 96 \\
\hline $\begin{array}{l}\text { Óxido de tântalo } \\
\text { de alta pureza }\end{array}$ & $\mathrm{Cu}, \mathrm{Fe}, \mathrm{Mn}, \mathrm{Na}$ e $\mathrm{Zn}$ & $\begin{array}{l}\text { Calibração com solução aquosa } \\
\text { Massa de amostra entre } 0,4 \text { e } 40 \mathrm{mg}\end{array}$ & 97 \\
\hline $\begin{array}{l}\text { Óxido de titânio, } \\
\text { óxido de nióbio e } \\
\text { óxido de zircônio }\end{array}$ & $\mathrm{Si}$ & $\begin{array}{l}\text { Calibração com solução aquosa } \\
\text { Modificador químico: } \mathrm{Pd}\left(\mathrm{NO}_{3}\right)_{2}+\mathrm{Mg}\left(\mathrm{NO}_{3}\right)_{2}\end{array}$ & 98 \\
\hline
\end{tabular}


Tabela 2. continuação

\begin{tabular}{|c|c|c|c|}
\hline Amostra & Elementos & Comentários & Ref. \\
\hline Óxido de zircônio & $\begin{array}{l}\mathrm{Cd}, \mathrm{Co}, \mathrm{Cr}, \mathrm{Cu}, \mathrm{Fe} \\
\mathrm{Li}, \mathrm{Mn}, \mathrm{Ni} \text { e } \mathrm{Zn}\end{array}$ & $\begin{array}{l}\text { Calibração com solução aquosa } \\
\text { Tamanho de partícula inferior a } 1 \mu \mathrm{m} \\
\text { Massa de amostra entre } 0,5 \text { e } 40 \mathrm{mg}\end{array}$ & 99 \\
\hline Ouro & $\mathrm{Si}$ & $\begin{array}{l}\text { Comparação dos resultados por suspensões em ICP-OES } \\
\text { Massa de amostra entre } 0,2 \text { e } 0,5 \mathrm{mg} \\
\text { Uso de } \mathrm{N}_{2} \text { para a remoção de água na etapa de secagem }\end{array}$ & 100 \\
\hline Pentóxido de nióbio & $\begin{array}{l}\mathrm{Al}, \mathrm{Co}, \mathrm{Cr}, \mathrm{Cu}, \mathrm{Fe}, \mathrm{K} \\
\mathrm{Mg}, \mathrm{Mn}, \mathrm{Na}, \mathrm{Ni} \text { e } \mathrm{Zn}\end{array}$ & $\begin{array}{l}\text { Calibração com solução aquosa } \\
\text { Adição de metano para remoção da matriz }\end{array}$ & 101 \\
\hline Pó de Mo metálico e & $\mathrm{Cu}, \mathrm{K}, \mathrm{Mg}, \mathrm{Mn}, \mathrm{Na}$ e $\mathrm{Zn}$ & Comparação dos resultados por RNAA e GDMS & 102 \\
\hline Silicato de molibdênio & & $\begin{array}{l}\text { Eliminação de molibdênio durante o programa de } \\
\text { aquecimento do forno de grafite }\end{array}$ & \\
\hline Prata de alta pureza & $\mathrm{Au}, \mathrm{Pd}$ e Pt & $\begin{array}{l}\text { Calibração com solução aquosa e com material sólido "in house" } \\
\text { Adição de } 15 \mu \mathrm{L} \text { de } \mathrm{HNO}_{3} 25 \% \mathrm{v} / \mathrm{v} \\
\text { Massa de amostra entre } 0,1 \text { e } 0,5 \mathrm{mg}\end{array}$ & 103 \\
\hline Tântalo de alta pureza & $\mathrm{Cu}, \mathrm{Fe}, \mathrm{K}, \mathrm{Mg}, \mathrm{Mn}, \mathrm{Na}$ e $\mathrm{Zn}$ & $\begin{array}{l}\text { Calibração com solução aquosa } \\
\text { Tamanho de partículas inferiores a } 70 \mu \mathrm{m} \\
\text { Massa de amostra entre } 0,1 \text { e } 8 \mathrm{mg}\end{array}$ & 104 \\
\hline Titânio de alta pureza & $\begin{array}{c}\mathrm{Al}, \mathrm{Ca}, \mathrm{Co}, \mathrm{Cr}, \mathrm{Cu}, \mathrm{Fe}, \mathrm{K} \\
\mathrm{Mg}, \mathrm{Mn}, \mathrm{Na}, \mathrm{Ni}, \mathrm{Pb}, \mathrm{Sn} \text { e } \mathrm{Zn}\end{array}$ & $\begin{array}{l}\text { Adição de C em pó } \\
\text { Adição de } 5 \% \text { v/v } \mathrm{HNO}_{3} \text { e } 2 \% \text { v/v } \mathrm{HF} \\
\text { Massa da amostra } 0,2 \text { e } 5 \mathrm{mg}\end{array}$ & 105 \\
\hline $\begin{array}{l}\text { Trióxido de tungstênio } \\
\text { e óxido de tungstênio azul }\end{array}$ & $\begin{array}{l}\mathrm{Ca}, \mathrm{Co}, \mathrm{Cr}, \mathrm{Cu}, \mathrm{Fe} \\
\mathrm{K}, \mathrm{Mg}, \mathrm{Na}, \mathrm{Ni} \text { e } \mathrm{Zn}\end{array}$ & $\begin{array}{l}\text { Calibração com solução aquosa } \\
\text { Tamanho das partículas entre } 0,5 \text { e } 2 \mu \mathrm{m}\left(\mathrm{WO}_{3}\right) \\
\text { e } 5 \text { a } 150 \mu \mathrm{m}\left(\mathrm{WO}_{3-\mathrm{X}}\right)\end{array}$ & 106 \\
\hline Tungstênio de alta pureza & $\begin{array}{l}\mathrm{Ca}, \mathrm{Co}, \mathrm{Cr}, \mathrm{Cu}, \mathrm{Fe}, \mathrm{K} \\
\mathrm{Mg}, \mathrm{Mn}, \mathrm{Na}, \mathrm{Ni} \text { e } \mathrm{Zn}\end{array}$ & $\begin{array}{l}\text { Adição de } \mathrm{H}_{2} \text { na etapa de pirólise } \\
\text { Calibração com solução aquosa } \\
\text { Massa de amostra entre } 0,15 \text { e } 100 \mathrm{mg}\end{array}$ & 107 \\
\hline
\end{tabular}

Tabela 3. Aplicação da SS ETAAS na análise de amostras ambientais

\begin{tabular}{|c|c|c|c|}
\hline Amostra & Elementos & Comentários & Ref. \\
\hline Água de rio e mar & $\mathrm{Cr}$ & $\begin{array}{l}\text { Co-precipitação com complexo Pd/8-quinolinol/ácido tânico } \\
\text { Massas de amostra } 0,1 \text { e } 1,5 \mathrm{mg}\end{array}$ & 108 \\
\hline Águas subterrâneas & $\mathrm{Cu}, \mathrm{Cd}$ & Massa de amostra de aproximadamente $5 \mathrm{mg}$ & 109 \\
\hline Água do mar & As (III), As (V) & Análise após coprecipitação com APDC e Ni-PDC & 110 \\
\hline $\begin{array}{l}\text { Amostras ambientais } \\
\text { (material particulado) }\end{array}$ & $\mathrm{Cd}, \mathrm{Cr}, \mathrm{Ni}(\mathrm{V})$ & $\begin{array}{l}\text { Calibração com solução aquosa } \\
\text { Modificadores químicos } \mathrm{NH}_{4} \mathrm{H}_{2} \mathrm{PO}_{4}, \mathrm{Mg}\left(\mathrm{NO}_{3}\right)_{2} \\
\text { Uso de Triton X-100 }\end{array}$ & 111 \\
\hline $\begin{array}{l}\text { Amostras geológicas } \\
\text { (diferentes solos } \\
\text { e rochas) }\end{array}$ & $\mathrm{As}, \mathrm{Cd}, \mathrm{Pb}, \mathrm{Hg}, \mathrm{Sb}$ e $\mathrm{Se}$ & $\begin{array}{l}\text { Massa da amostra entre } 1 \text { e } 5 \mathrm{mg} \\
\text { Modificadores químicos } \mathrm{H}_{3} \mathrm{PO}_{4}, \mathrm{Mg}\left(\mathrm{NO}_{3}\right)_{2}, \mathrm{Ni}\left(\mathrm{NO}_{3}\right)_{2} \\
\text { Amostras diluídas com grafite para eliminar picos duplos ou } \\
\text { interferências causadas por amostras vítreas }\end{array}$ & 112 \\
\hline Amostras geológicas & $\mathrm{Cd}, \mathrm{Cr}, \mathrm{Cu}, \mathrm{Ni}, \mathrm{Pb}, \mathrm{Zn}$ & $\begin{array}{l}\text { Calibração com solução aquosa } \\
\text { Avaliação da homogeneidade das amostras } \\
\text { Massa da amostra entre } 0,05 \text { e } 1,5 \mathrm{mg}\end{array}$ & 113 \\
\hline Amostras geológicas & $\mathrm{Hg}$ & Massa de amostra de 30 e $400 \mathrm{mg}$ & 114 \\
\hline Ar atmosférico & $\mathrm{Pb}$ & $\begin{array}{l}\text { Depósito de material particulado sobre a } \\
\text { plataforma por um determinado tempo }\end{array}$ & 115 \\
\hline Carvão & $\mathrm{Cd}$ & $\begin{array}{l}\text { Calibração com solução aquosa } \\
\text { Modificador químico: } 400 \mu \mathrm{g} \mathrm{Ir} \\
\text { Massa de amostra entre } 0,6 \text { e } 1,2 \mathrm{mg}\end{array}$ & 116 \\
\hline Carvão & $\mathrm{Hg}$ & $\begin{array}{l}\text { Calibração com solução aquosa } \\
\text { Modificador químico: Pd eletrodepositado } \\
\text { Tamanho de partículas }<50 \mu \mathrm{m}\end{array}$ & 47 \\
\hline Carvão & $\mathrm{Pb}$ & $\begin{array}{l}\text { Calibração com solução aquosa } \\
\text { Massa de amostra entre } 0,1 \text { e } 1,0 \mathrm{mg}\end{array}$ & 117 \\
\hline Carvão mineral & $\mathrm{Cd}, \mathrm{Cu}$ e $\mathrm{Pb}$ & $\begin{array}{l}\text { Calibração com solução aquosa } \\
\text { Modificador químico: } \mathrm{Pd}\left(\mathrm{NO}_{3}\right)_{2}+\mathrm{Mg}\left(\mathrm{NO}_{3}\right)_{2} \mathrm{e} \mathrm{Ru}\end{array}$ & 118 \\
\hline Lodo & $\mathrm{Cu}, \mathrm{Pb}$ & $\begin{array}{l}\text { Calibração com solução aquosa } \\
\text { Massa de amostra de } 0,15 \mathrm{mg}(\mathrm{Cu}) \text { e } 2 \mathrm{mg}(\mathrm{Pb})\end{array}$ & 119 \\
\hline
\end{tabular}


Tabela 3. continuação

\begin{tabular}{|c|c|c|c|}
\hline Amostra & Elementos & Comentários & Ref. \\
\hline Materiais ambientais & $\mathrm{Hg}$ & $\begin{array}{l}\text { Calibração com solução aquosa } \\
\text { Modificador químico permanente: Ir, Pd, Rh, Ru } \\
\text { Massa de amostra entre } 0,035 \text { e } 0,32 \mathrm{mg}\end{array}$ & 120 \\
\hline $\begin{array}{l}\text { Material particulado } \\
\text { industrial }\end{array}$ & $\mathrm{Cd}, \mathrm{Cr}, \mathrm{Cu}, \mathrm{Ni}, \mathrm{Pb}$ & $\begin{array}{l}\text { Material particulado coletado em filtro } \\
\text { Filtro cortado em círculos de diâmetros de } 1 \mathrm{~mm} \text { e } \\
\text { analisados por SS ETAAS } \\
\text { Modificador químico: } \mathrm{Pd}\left(\mathrm{NO}_{3}\right)_{2} \\
\text { Calibração com solução aquosa }\end{array}$ & 121 \\
\hline Sedimento de rio & $\mathrm{Cd}, \mathrm{Pb}$ e $\mathrm{Cr}$ & $\begin{array}{l}\text { Massa de amostra } 20 \text { e } 400 \mu \mathrm{g} \\
\text { Calibração com solução aquosa }\end{array}$ & 122 \\
\hline $\begin{array}{l}\text { Sedimento de rio e } \\
\text { sedimento marinho }\end{array}$ & $\mathrm{Tl}$ & $\begin{array}{l}\text { Tamanho de partículas inferiores a } 50 \mu \mathrm{m} \\
\text { Calibração com solução aquosa }\end{array}$ & 123 \\
\hline Sedimentos, solo, rochas & $\mathrm{Cu}, \mathrm{Ni}, \mathrm{Pb}$ e $\mathrm{Zn}$ & Calibração com solução aquosa & 124 \\
\hline Solo & $\mathrm{Hg}$ & $\begin{array}{l}\text { Calibração com solução aquosa; } \\
\text { Modificador químico: } \mathrm{KMnO}_{4}\end{array}$ & 125 \\
\hline Solo & Co, $\mathrm{Zn}$ & $\begin{array}{l}\text { Calibração com solução aquosa; } \\
\text { Massa de amostra entre } 5 \text { e } 10 \mathrm{mg}\end{array}$ & 126 \\
\hline
\end{tabular}

\section{AGRADECIMENTOS}

À Fundação de Amparo à Pesquisa do Estado de São Paulo pelo suporte financeiro e pelas bolsas concedidas à C. S. Nomura e C. S. Silva, e ao Conselho Nacional de Desenvolvimento Científico e Tecnológico (CNPq) pelo suporte financeiro e pela bolsa de produtividade concedida a $\mathrm{P}$. V. Oliveira.

\section{REFERÊNCIAS}

1. Sulcek, Z.; Povondra, P.; Methods of Decomposition in Inorganic Analysis, $1^{\text {st }}$ ed., CRC Press: Boca Raton, 1989.

2. Anderson, R.; Sample Pretreatment and Separation, Chichester, John Wiley \& Sons: New York, 1991.

3. Kingston, H. M.; Haswell, S. J.; Microwave-Enhanced Chemistry: Fundamentals, Sample Preparation, $1^{\text {st }}$ ed., American Chemical Society: Ohio, 1997.

4. Welz, B.; Sperling, M.; Atomic Absorption Spectrometry - Third, Completely Revised Edition, Wiley-VCH Verlag GmbH: Weinheim, 1999.

5. Jackson, K. W.; Electrothermal atomization for analytical atomic spectrometry, John Willey \& Sons: New York, 1999.

6. Kurfüst, U.; Solid Sample Analysis, Springer-Verlag: Berlin-Heidelberg, 1998.

7. Bendicho, C.; de Loos-Vollebregt, M. T. C.; J. Anal. At. Spectrom. 1991, 6,353 .

8. Vale, M. G. R.; Oleszczuk, N.; Santos, W. N. L.; Appl. Spectrosc. Rev. 2006, $41,377$.

9. Cal-Prieto, M. J.; Felipe-Sotelo, M.; Carlosena, A.; Andrade, J. M.; LopezMahia, P.; Muniategui, S.; Prada, D.; Talanta 2002, 56, 1

10. Magalhães, C. E. C.; Arruda, M. A. Z.; Quim. Nova 1998, 21, 459.

11. Belarra, M. A.; Resano, M.; Vanhaecke, F.; Moens, L.; Trends Anal. Chem. 2002, 21, 828 .

12. L' Vov, B. V.; Spectrochim. Acta, Part B 1984, 39, 149

13. Massmann, H.; Spectrochim. Acta, Part B 1968, 23, 215.

14. Friese, K. C.; Krivan, V.; Spectrochim. Acta, Part B 1998, 53, 1069.

15. Welz, B.; Becker-Ross, H.; Florek, S.; Heitmann, U.; High-Resolution Continuum Source AAS, Wiley-VCH Verlag GMbH: Weinheim, 2005.

16. Resano, M.; Belarra, M. A.; Castillo, J. R.; Vanhaecke, F.; J. Anal. At. Spectrom. 2000, 15, 1383.

17. Lucker, E.; Schuierer, O.; Spectrochim. Acta, Part B 1996, 51, 201.

18. Uchino, E.; Jin, K.; Tsuzuki, T.; Inoue, K.; Analyst 1987, 112, 291.

19. Harrison, S. H.; LaFleur, P. D.; Anal. Chem. 1975, 47, 1685.

20. Yanan, C.; Fresenius J. Z. Anal. Chem. 1987, 326, 705.

21. Nomura, C. S.; Silva, C. S.; Nogueira, A. R. A.; Oliveira, P. V.; Spectrochim. Acta, Part B 2005, 60, 673.

22. Zeisler, R.; Fresenius. J. Anal. Chem. 1998, 360, 376.

23. Iyengar, G. V.; Kasperek, K.; J. Radioanal. Nucl. Chem. 1977, 39, 301.

24. Koglin, D.; Backhaus, F.; Schladot, J. D.; Chemosphere 1997, 34, 2041
25. Santos, D.; Barbosa, F.; Tomazelli, A. C.; Krug, F. J.; Nóbrega, J. A.; Arruda, M. A. Z.; Anal. Bioanal. Chem. 2002, 373, 183.

26. Santos, D.; Barbosa, F.; Souza, S. S.; Krug, F. J.; J. Anal. At. Spectrom. 2003, 18, 939

27. Lima, E. C.; Krug, F. J.; Ferreira, A. T.; Barbosa Junior, F.; J. Anal. At. Spectrom. 1997, 12, 375.

28. Fuller, C. W.; Hetton, R. C.; Preston, B.; Analyst 1981, 106, 913.

29. Fajgelj, A.; Zeisler, R.; Fresenius. J. Anal. Chem. 1998, 360, 442.

30. Stoeppler, M.; Wolf, W. R.; Jenks, P. J., eds.; Reference Materials for Chemical Analysis - Certification, Avaiability, and Proper Usage, $1^{\text {st }}$ ed., Wiley-VCH Verlag GMbH: Weinheim, 2001.

31. Grant, R.; Grant C.; Grant and Hackh's Chemical Dictionary, $5^{\text {th }}$ ed., McGraw-Hill: Texas, 1987.

32. Pauwels, J.; Hofmann, C.; Vandecasteele, C.; Fresenius. J. Anal. Chem. 1994, 348, 418.

33. Rossbach, M.; Ostapczuck, P.; Emons, H.; Fresenius J. Anal. Chem. 1998, $360,380$.

34. Langmyhr, F. J.; Fresenius J. Anal. Chem. 1985, 322, 654.

35. Belarra, M. A.; Crespo, C.; Martínez-Garbayo, M. P.; Castillo, J. R.; Spectrochim. Acta, Part B 1997, 52, 1855.

36. Takada, K.; Hirokawa, K.; Fresenius J. Anal. Chem. 1982, 312, 109.

37. Kurfürst, U.; Pure Appl. Chem. 1991, 63, 1205.

38. Nomura, C. S.; Oliveira, P. V.; Quim. Nova 2006, 29, 234.

39. ISO Guia 35; Certification of Reference Materials General and Statistical Principles, ISSO: Geneva, 1989.

40. Ingamells, C. O.; Switzer, P.; Talanta 1973, 20, 547.

41. Kurfürst, U.; Pauwels, J.; Grobecker, K. H.; Stoeppler, M.; Muntau, H.; Fresenius J. Anal. Chem. 1993, 345, 112.

42. Berglund, M.; Baxter, D. C.; Spectrochim. Acta, Part B 1992, 47, E1567.

43. Gleisner, H.; Eichardt, K.; Welz, B.; Spectrochim. Acta, Part B 2003, 58, 1663.

44. Resano, M.; Aramendía, M.; García-Ruiz, E.; Crespo, C.; Belarra M. A.; Anal. Chim. Acta 2006, 571, 142.

45. Nomura, C. S.; Tese de Doutorado Universidade de São Paulo, Brasil, 2006.

46. Borges, D. L. G.; Silva, A. F.; Welz, B.; Curtius, A. J.; Heitmann, U.; J. Anal. At. Spectrom. 2006, 21, 763.

47. Maia, S. M.; Welz, B.; Ganzarolli, E.; Curtius, A. J.; Spectrochim. Acta, Part B 2002, 57, 473

48. Coskun, N.; Akman, S.; Spectrochim. Acta, Part B 2005, 60, 415.

49. Silva, A. F.; Lepri, F. G.; Borges, D. L. G.; Welz, B.; Curtius, A. J.; Heitmann, U.; J. Anal. At. Spectrom. 2006, 21, 1321.

50. Minami, H.; Zhang, Q.; Itoh, H.; Atsuya, I.; Microchem. J. 1994, 49, 126.

51. Belarra, M. A.; Resano, M.; Castillo, J. R.; J. Anal. At. Spectrom. 1998, 13, 489 .

52. Stupar, J.; Dolinsek, F.; Spectrochim. Acta, Part B 1996, 51, 665.

53. Koone, R. D.; Peters, C. A.; J. Anal. Toxicol. 1994, 18, 36.

54. Strübel, G.; Rzepka-Glinder, V.; Grobecker, K. H.; Jarrar, K.; Fresenius J. Anal. Chem.1990, 337, 316.

55. Strübel, G.; Rzepka-Glinder, V.; Grobecker, K. H.; Fresenius J. Anal. Chem. 1987, 328, 382 . 
56. Mohl, C.; Grobecker, K. H.; Stoeppler, M.; Fresenius J. Anal. Chem. 1987, $328,413$.

57. Lücker, E.; Rosopulo, A.; Kreuzer, W.; Fresenius J. Anal. Chem. 1991, 340, 234.

58. Lücker, E.; Fresenius J. Anal. Chem. 1992, 343, 386.

59. Schauenburg, H.; Weigert, P.; Fresenius J. Anal. Chem. 1991, 342, 950.

60. Chakrabarti, C. L.; Wan, C. C.; Li, W. C.; Spectrochim. Acta, Part B 1980 , $35,93$.

61. Lucker, E.; Konig, H.; Gabriel, W.; Fresenius J. Anal. Chem. 1992, 342, 941

62. Lücker, E.; Meuthen, J.; Kreuzer, W.; Fresenius J. Anal. Chem. 1993, 346, 1068.

63. Lücker, E.; Appl. Spectrosc. 1997, 51, 1031.

64. Lücker, E.; Gerbig, C.; Kreuzer, W.; Fresenius J. Anal. Chem. 1993, 346, 1062.

65. Dobrowolski, R.; Mierzwa, J.; Fresenius J. Anal. Chem. 1992, 344, 340

66. Atsuya, I.; Akatsuka, K.; Itoh, K.; Fresenius J. Anal. Chem. 1990, 337, 294.

67. Detcheva, A.; Grobecker, K. H.; Spectrochim. Acta, Part B 2006, 61, 454.

68. Byrd, E. D.; Butcher, D. J.; Spectrosc. Lett. 1993, 26, 1613.

69. Pauwels, J.; Kurfurst, U.; Grobecker, K. H.; Quevauviller, P.; Fresenius J. Anal. Chem. 1993, 345, 478.

70. Lücker, E; Thorius-Ehrler, S.; Fresenius J. Anal. Chem. 1993, 346, 1072.

71. Lücker, E.; J. Anal. At. Spectros 1999, 14, 583.

72. Lücker, E.; J. Anal. At. Spectrom. 1999, 14, 1731

73. Hofmann, C.; Vandercasteele, C.; Pauwels, J.; Fresenius J. Anal. Chem. 1992, 342, 936.

74. Hahn, E.; Hahn, K.; Mohl, C.; Stoepler, M.; Fresenius J. Anal. Chem. 1990, 337, 306.

75. Lücker, E.; Fresenius J. Anal. Chem. 1997, 358, 848.

76. Lücker, E.; Rosopulo, A.; Kreuzer, W.; Fresenius J. Anal. Chem. 1987, 328 , 370.

77. Bagschik, U.; Quack, D.; Stoeppler, M.; Fresenius J. Anal. Chem. 1990, $338,386$.

78. Akatsuka, K.; Atsuya, I.; Fresenius J. Anal. Chem. 1989, 335, 200.

79. Coskun, N.; Akman, S.; Talanta 2004, 64, 496.

80. Pauwels, J.; Hofmann, C.; Grobecker, K.; Fresenius J. Anal. Chem. 1993, 345,475 .

81. Pauwels, J.; Lambert, A.; De Biève, P.; Grobecker, K. H.; Bauspiess, C.; Fresenius J. Anal. Chem. 1994, 349, 409.

82. Resano, M.; Aramendia, M.; Volynsky, A. B.; Belarra, M. A.; Spectrochim. Acta, Part B 2004, 59, 523.

83. Belarra, M. A.; Resano, M.; Rodríguez, S.; Urchaga, J.; Spectrochim. Acta, Part B 1999, 54, 787

84. Belarra, M. A.; Belategui, I.; Lavilla, I.; Anzano, J. M.; Castillo, J. R.; Talanta 1998, 46, 1265.

85. Nowka, R.; Marr, I. L.; Ansari, T. M.; Muller, H.; Fresenius J. Anal. Chem 1999, 364, 533 .

86. Scaffer, U.; Krivan, V.; Fresenius J. Anal. Chem. 2001, 371, 859.

87. Nowka, R.; Muller, H.; Fresenius J. Anal. Chem. 1997, 359, 132.

88. Pauwels, J.; De Angelis, L.; Peetermans, F.; Ingelbrecht, C.; Fresenius J. Anal. Chem. 1990, 337, 290.

89. Schothhorst, R. C.; Géron, H. M. A.; Spitsbergen, D.; Herber, R. F.; Fresenius J. Anal. Chem. 1987, 328, 393.

90. Schron, W.; Detcheva, A.; Dressler, B.; Danzer, K.; Fresenius J. Anal. Chem. 1998, 361, 106.
91. Scaffer, U.; Krivan, V.; Fresenius J. Anal. Chem. 2001, 371, 859.

92. Awad, N. A.; Jasim, F.; Microchem. J. 1989, 40, 187.

93. Irwin, R.; Mikkelsen, A.; Michel, R. G.; Dougherty, J. P.; Preli, F. R.; Spectrochim. Acta, Part B 1990, 45, 903.

94. Schron, W.; Liebmann, A.; Nimmerfall, G.; Fresenius J. Anal. Chem. 2000, 366, 79.

95. Docekal, B.; Spectrochim. Acta, Part B, 1998, 53, 427.

96. Lucic, M.; Krivan, V.; J. Anal. At. Spectrom. 1998, 13, 1133.

97. Friese, K. C.; Krivan, V.; Schuierer, O.; Spectrochim. Acta, Part B 1996, $51,1223$.

98. Dong, H.; Krivan, V.; Spectrochim. Acta, Part B 2001, 56, 1645.

99. Krivan, V.; Janickova, P.; Anal. Bioanal. Chem. 2005, 382, 1949.

100. Brown, G. N.; Styris, D. L.; J. Anal. At. Spectrom. 1995, 10, 527.

101. Huang, M. D.; Krivan, V.; Fresenius J. Anal. Chem. 2000, 368, 227

102. Docekal, B.; Krivan, V.; Spectrochim. Acta, Part B 1995, 50, 517.

103. Hinds, M. W.; Spectrochim. Acta, Part B 1993, 48, 435.

104. Friese, K. C.; Krivan, V.; Spectrochim. Acta, Part B 1998, 53, 1069.

105. Krivan, V.; Dong, H. M.; Anal. Chem. 1998, 70, 5312.

106. Hornung, M.; Krivan, V.; Spectrochim. Acta, Part B 1999, 54, 1177.

107. Hornung, M.; Krivan, V.; Anal. Chem. 1998, 70, 3444.

108. Zhang, Q.; Minami, H.; Inoue, S.; Atsuya, I.; Anal. Chim. Acta 1999, 401, 277.

109. Freedman, Y. E.; Ronen, D.; Long, G. L.; Environ. Sci. Technol. 1996, 30, 2270.

110. Zhang, Q.; Minami, H.; Inoue, S.; Atsuya, I.; Anal. Chim. Acta 2004, 508, 99.

111. Schlemmer, G.; Welz, B.; Fresenius J. Anal. Chem. 1987, 328, 405.

112. Dekersabiec, A. M.; Benedetti, M. F.; Fresenius J. Anal. Chem. 1987, 328, 342.

113. Nimmerfall, G.; Schron, W.; Fresenius J. Anal. Chem. 2001, 370, 760.

114. Hall, G. E. M.; Pelchat, P.; Analyst 1997, 122, 921.

115. Low, P. S.; Hsu, G. J.; Fresenius J. Anal. Chem. 1990, 337, 299.

116. Silva, A. F.; Borges, D. L. G.; Lepri, F. G.; Welz, B.; Curtius, A. J.; Heitmann, U.; Anal. Bioanal. Chem. 2005, 382, 1835.

117. Borges, D. L. G.; Silva, A. F.; Curtius, A. J.; Welz, B.; Heitmann, U.; Microchim. Acta 2006, 154, 101.

118. Vale, M. G. R.; Silva, M. M.; Welz, B.; Lima, E. C.; Spectrochim. Acta. Part B 2001, 54, 1859.

119. Belarra, M. A.; Crespo, C.; Resano, M.; Castillo, J. R.; Spectrochim. Acta, Part B 2000, 55, 865.

120. Silva, A. F.; Welz, B.; Curtius, A. J.; Spectrochim. Acta, Part B 2002, 57, 2031.

121. Almeida, A. A.; Lima, J. L. F. C.; At. Spectrosc. 1995, 16, 261.

122. Sahuquillo, A.; Rauret, G.; Rehnert, A.; Muntau, H.; Anal. Chim. Acta 2003, $476,15$.

123. Vale, M. G. R.; Silva, M. M.; Welz, B.; Nowka, R.; J. Anal. At. Spectrom. 2002, 17, 38

124. Schron, W.; Liebmann, A.; Nimmerfall, G.; Fresenius J. Anal. Chem. 2000, 366, 79.

125. Resano, M.; García-Ruiz, E.; Aramendia, M.; Belarra, M. A.; J. Anal. At. Spectrom. 2005, 20, 1374

126. Belarra, M. A.; Crespo, C.; Martinez-Gasbayo, M. P.; Resano, M.; Spectrochim. Acta, Part B 2003, 58, 1847. 